\title{
Certain Bilateral Generating Relations for a Class of Generalized Hypergeometric Functions of Two Variables
}

\author{
B.Satyanarayana $^{1, *}$, N. Srimannarayana ${ }^{2}$, Y. Pragathi Kumar ${ }^{1}$ \\ ${ }^{1}$ Department of Mathematics, Acharya Nagarjuna University, Nagarjuna Nagar-522 510, Andhra Pradesh, India \\ ${ }^{2}$ Department of Science and Humanities, RISE Gandhi Group of Institutions, Ongole -523 272, Andhra Pradesh, India \\ *Corresponding Author: drbsn63@yahoo.co.in
}

Copyright (C) 2014 Horizon Research Publishing All rights reserved.

Abstract In [10] we defined and studied a class of generalized hypergeometric functions $B_{n}^{(\alpha, \beta)}(x, y, w)$. In this paper an attempt has been made to obtain some bilateral generating relations with $B_{n}^{(\alpha, \beta)}(x, y, w)$. Each result is followed by its applications to the classical orthogonal polynomials.

Keywords Bilateral Generating Relations, Generalized Hypergeometric Functions, Classical Orthogonal Polynomials

\section{Introduction}

In the previous paper [10], we introduced a class of generalized hypergeometric functions $B_{n}^{(\alpha, \beta)}(x, y, w)$ defined as follows:

$$
\begin{gathered}
B_{n}^{(\alpha, \beta)}(x, y, w)=\frac{\Gamma(n+\alpha+1) \Gamma(n+\beta+1)}{(n !)^{2}} \times \\
\sum_{r=0}^{n} \frac{(-1)^{r} y^{[r w]}}{r ! \Gamma(n+\alpha-r+1) \Gamma(\beta+r+1)} J_{n-r}^{\alpha}(x, w)
\end{gathered}
$$

where $J_{n}^{\alpha}(x, w)$ is modified Jacobi polynomial (see Parihar and Patel [6] and also see Lahiri and Satyanarayana [3]-[5]). We also derived the following relation

$$
\begin{gathered}
B_{n}^{(\alpha, \beta)}(x, y, w)=\frac{(1+\alpha)_{n}(1+\beta)_{n}}{(n !)^{2}} \times, \\
F_{-: 1 ; 1}^{1: 1 ; 1}\left[\begin{array}{c}
-n:-\frac{y}{w} ; \frac{x}{w} ;-w, w \\
--: 1+\beta ; 1+\alpha ;
\end{array}\right] \quad \text { (1.2) }
\end{gathered}
$$

Taking the limit $w \rightarrow 0$ in (1.1), we obtain

$$
\lim _{w \rightarrow 0} B_{n}^{(\alpha, \beta)}(x, y, w)=L_{n}^{(\alpha, \beta)}(x, y),
$$

where $L_{n}^{(\alpha, \beta)}(x, y)$ is Laguerre polynomial of two variables [7].

In Satyanarayana [9] ( also see [5, p.326(1.8)] defined generalized hypergeometric functions $I_{n ; \lambda ;\left(b_{q}\right)}^{\alpha ; \mu ;\left(a_{p}\right)}(x, w)$ and also proved that $[9, \mathrm{p} .65(3.3 .3)]$

$$
\begin{gathered}
\sum_{n=0}^{\infty}\left(\begin{array}{c}
n+r \\
r
\end{array}\right) \frac{(\rho+r)_{n}}{(1+\alpha+r)_{n}} I_{n+r ; \lambda ;\left(b_{q}\right)}^{\alpha ; \mu ;\left(a_{p}\right)}(x, w) t^{n} \\
=(1-t)^{-\rho-r}\left(\begin{array}{c}
\alpha+r \\
r
\end{array}\right) \\
F^{(3)}\left[\begin{array}{c}
\left(a_{p}\right):: \frac{x}{w}-\mu+1 ;--;--; \\
\left(b_{q}\right):: 1+\alpha ;--;--; \\
\rho+r ;-r ; \frac{-x}{w}+\lambda-\frac{w t}{1-t}, w, w \\
--;--;--;
\end{array}\right]
\end{gathered}
$$

where $F^{(3)}$ is generalized hypergeometric functions of three variables (see Srivastava and Karlsson [11]).

In particular for $\lambda=0$ and $\mu=1$, we have

$$
I_{n ; 0 ;\left(a_{p}\right)}^{\alpha ; 1 ;\left(a_{p}\right)}(x, w)=(1-w)^{\frac{x}{w}} J_{n}^{\alpha}(x, w),
$$

where $J_{n}^{\alpha}(x, w)$ is modified Jacobi polynomial (see Parihar and Patel [6]) and

$$
\lim _{w \rightarrow 0} I_{n ; \lambda ;\left(a_{p}\right)}^{\alpha ; \mu ;\left(a_{p}\right)}(x, w)=e^{-x} L_{n}^{\alpha}(x),
$$

where $L_{n}^{\alpha}(x)$ is Laguerre polynomial [8].

The following definitions and results given by Rainville [ 8 , 
p.302] Gottlieb polynomial

$$
\phi_{n}(x ; \lambda)=e_{2}^{-n \lambda} F_{1}\left(-n,-x ; 1 ; 1-e^{\lambda}\right),
$$

Generalized Sylvester polynomial

$$
f_{n}(x ; a)=\frac{(a x)^{n}}{n !}{ }_{2} F_{0}\left(-n, x ;-;-\frac{1}{a x}\right)
$$

Agarwal and Manocha $[1$, p.1372 (2.2)(5.5)]

$$
\begin{gathered}
\sum_{n=0}^{\infty}\left(\begin{array}{c}
n+k \\
k
\end{array}\right) \phi_{n+k}(x ; \lambda) t^{n}=(1-t)^{x-k} \times \\
\left(1-t e^{-\lambda}\right)^{-x-1} \phi_{k}\left(x ; \log _{e}\left(\frac{e^{\lambda}-t}{1-t}\right)\right)
\end{gathered}
$$

and

$$
\begin{gathered}
\sum_{n=0}^{\infty}\left(\begin{array}{c}
n+k \\
k
\end{array}\right) f_{n+k}(x ; a) t^{n}=(1-t)^{-x-k} \times \\
e^{a x t} f_{k}(x ; a(1-t))
\end{gathered}
$$

\section{Main Results}

\section{Bilateral generating relations}

We have derived the following bilateral generating relations for the class of generalized hypergeometric functions $B_{n}^{(\alpha, \beta)}(x, y, v)$ :

$$
\begin{aligned}
& \sum_{n=0}^{\infty} \frac{(\rho)_{n}(n !)^{2}}{\left[(1+\alpha)_{n}\right]^{2}(1+\beta)_{n}} \times \\
& B_{n}^{(\alpha, \beta)}{ }_{(x, y, v)} I_{n ; \lambda ;\left(b_{q}\right)}^{\alpha ; \mu ;\left(a_{p}\right)}(x, w) t^{n} \\
& =(1-t)^{-\rho} F_{q+2: 0 ; 0 ; 0 ; 1 ; 1}^{p+2: 0 ; 0 ; 1 ; 2}\left[\begin{array}{l}
{\left[\left(a_{p}\right): 1,1,1,0,1\right],} \\
{\left[\left(b_{q}\right): 1,1,1,0,1\right],}
\end{array}\right. \\
& {[\rho: 1,1,0,1,1],\left[\frac{x}{w}-\mu+1: 1,1,0,0,1\right]:} \\
& {[1+\alpha: 1,1,0,0,1],[\rho: 0,1,0,1,1,]:} \\
& -;-;-\frac{x}{w}+\lambda ; \rho,-\frac{y}{v} ; \\
& -;-;-; 1+\beta \text {; }
\end{aligned}
$$

$$
\left.\frac{\frac{x}{v}}{1+\alpha} ; \frac{-w t}{1-t},-w, w, \frac{v t}{1-t}, w v\right]
$$

Where $\mathrm{F}$ is a generalized Lauricella hypergeometric function of 5 variables.

$$
\begin{gathered}
\sum_{n=0}^{\infty} \frac{(n !)^{2}}{(1+\alpha)_{n}(1+\beta)_{n}} B_{n}^{(\alpha, \beta)}(x, y, v) \times \\
\phi_{n}(x ; \lambda) t^{n} \\
=(1-t)^{x}\left(1-t e^{-\lambda}\right)^{-x-1} \times \\
F_{2: 0 ; 0 ; 1}^{3: 0 ; 1}\left[\begin{array}{c}
{[1: 1,1,1],\left[-\frac{y}{v}: 1,1,0\right],[-x: 1,0,1]:} \\
{[1+\beta: 1,1,1],[1: 1,0,1]:} \\
\left.-;-; x / v ; t_{1}, t_{2}, t_{3}\right] \\
-;-; 1+\alpha ;
\end{array}\right.
\end{gathered}
$$

Where $\mathrm{F}$ is a generalized Lauricella hypergeometric function of 3 variables.

$$
\begin{gathered}
t_{1}=\frac{v\left(e^{\lambda}-1\right) t}{\left(e^{\lambda}-t\right)(1-t)}, t_{2}=\frac{v t}{e^{\lambda}-t} \\
t_{3}=\frac{v^{2}\left(e^{\lambda}-1\right) t}{\left(e^{\lambda}-1\right)(1-t)} . \\
\sum_{n=0}^{\infty} \frac{(n !)^{2}}{(1+\alpha)_{n}(1+\beta)_{n}} B_{n}^{(\alpha, \beta)}(x, y, v) \times \\
f_{n}(x ; a) t^{n}=(1-t)^{-x} e^{a x t} F_{1: 0 ; 0 ; 1}^{2: 0 ; 0 ; 1}\left[\frac{-y}{v}: 1,1,1\right], \\
\left.[x: 1,0,1]:-;-; \frac{x}{v} ;\left(\frac{v t}{1-t}\right)^{n}, v \operatorname{tax},\left(\frac{-v^{2} t}{1-t}\right)\right] \\
-\quad:-;-; 1+\alpha ; 1,1,1],
\end{gathered}
$$

Proof of (2.1). From (2.1), we have

$$
\begin{aligned}
& \sum_{n=0}^{\infty} \frac{(\rho)_{n}(n !)^{2}}{\left[(1+\alpha)_{n}\right]^{2}(1+\beta)_{n}} \times \\
& B_{n}^{(\alpha, \beta)}{ }_{(x, y, v)} I_{n}^{\alpha}(x, w) t^{n}
\end{aligned}
$$




$$
\begin{gathered}
=\sum_{n=0}^{\infty} \sum_{s=0}^{n-r} \sum_{r=0}^{n} \frac{(\rho)_{n}}{(1+\alpha)_{n}} \frac{(-n)_{r+s}\left(-\frac{y}{v}\right)_{r}}{(1+\alpha)_{S}(1+\beta)_{r}} \times \\
\frac{\left(\frac{x}{v}\right)_{S}}{r !} \frac{(-v)^{r}}{s !} I_{n}^{\alpha}(x, w) t^{n} \\
=\sum_{s=0}^{n} \sum_{r=0}^{\infty} \frac{(-n)_{S}(\rho)_{r}\left(-\frac{y}{v}\right)_{r}\left(\frac{x}{v}\right)_{s}(v t)^{r} v^{s}}{(1+\alpha)_{r}(1+\alpha)_{S}(1+\beta)_{r} s !} \times \\
\sum_{n=0}^{\infty}\left(\begin{array}{l}
n+r \\
r
\end{array}\right) \frac{(\rho+r)_{n}}{(1+\alpha+r)_{n}} I_{n+r}^{\alpha}(x, w) t^{n}
\end{gathered}
$$

By using (1.4), we get

$$
\begin{aligned}
& =\sum_{s=0}^{n} \frac{(-n)_{S}\left(\frac{x}{v}\right)_{S} v^{S}}{(1+\alpha)_{S} s !} \sum_{r=0}^{\infty} \frac{\left(-\frac{y}{v}\right)_{r}(v t)^{r}(\rho)_{r}}{(1+\alpha)_{r}(1+\beta)_{r}} \\
& (1-t)^{-\rho-r}\left(\begin{array}{l}
\alpha+r \\
r
\end{array}\right) F^{(3)}\left[\begin{array}{l}
\left(a_{p}\right):: \\
\left(b_{q}\right)::
\end{array}\right. \\
& \left.\begin{array}{c}
\frac{x}{w}-\mu+1 ;-;-; \rho+r ;-r ;-\frac{x}{w}+\lambda ; \frac{-w t}{1-t}, w, w \\
1+\alpha ;-;-;-;-;-\quad ;
\end{array}\right] \\
& =(1-t)^{-\rho} \sum_{m, k, n, r, s=0}^{\infty} \frac{\left(a_{p}\right)_{m+n+k+s}}{\left(b_{q}\right)_{m+n+k+s}} \times \\
& \frac{(\rho)_{m+n+r+s}\left(\frac{x}{w}-\mu+1\right)_{m+n+s}}{(1+\alpha)_{m+n+s}(\rho)_{n+r+s}} \times \\
& \frac{\left(-\frac{x}{w}+\lambda\right)_{k}\left(-\frac{y}{v}\right)_{r}(\rho)_{r}\left(\frac{x}{v}\right)_{s}}{(1+\alpha)_{S}(1+\beta)_{r}} \times \\
& \left(\frac{-w t}{1-t}\right)^{m} \frac{(-w)^{n} w^{k}}{m ! n ! k !} \frac{\left(\frac{v t}{1-t}\right)^{r}}{r !} \frac{(w v)^{s}}{s !} \\
& =(1-t)^{-\rho} F_{q+2: 0 ; 0 ; 0 ; 1 ; 1}^{p+2: 0 ; 0 ; 1 ; 1}\left[\begin{array}{l}
{\left[\left(a_{p}\right): 1,1,1,0,1\right],} \\
{\left[\left(b_{q}\right): 1,1,1,0,1\right],}
\end{array}\right.
\end{aligned}
$$

$$
\begin{gathered}
{[\rho: 1,1,0,1,1],\left[\frac{x}{w}-\mu+1: 1,1,0,0,1\right]:} \\
{[1+\alpha: 1,1,0,0,1],[\rho: 0,1,0,1,1,]:} \\
-;-;-\frac{x}{w}+\lambda ; \rho,-\frac{y}{v} ; \frac{x}{v} \\
-;-;-; 1+\beta ; 1+\alpha \\
\left.\frac{-w t}{1-t},-w, w, \frac{v t}{1-t}, w v\right]
\end{gathered}
$$

Hence complete the proof of (2.1).

\section{Applications.}

(i) By setting $\mathrm{p}=\mathrm{q}, \mathrm{a}_{\mathrm{j}}=\mathrm{b}_{\mathrm{j}}, \mathrm{j}=1,2 \ldots \mathrm{p}, \mu=1$ and $\lambda=0$ in (2.1), we get

$$
\begin{aligned}
& \sum_{n=0}^{\infty} \frac{(\rho)_{n}(n !)^{2}}{\left[(1+\alpha)_{n}\right]^{2}(1+\beta)_{n}} \times \\
& B_{n}^{(\alpha, \beta)}{ }_{(x, y, v)} J_{n}^{\alpha}(x, w) t^{n}=(1-t)^{-\rho} \times \\
& F_{2: 0 ; 0 ; 1 ; 1}^{2: 0 ; 0 ; 2 ; 1}\left[\begin{array}{c}
{[\rho: 1,1,1,1],\left[\frac{x}{w}: 1,1,0,1\right]:} \\
{[1+\alpha: 1,1,0,1],[\rho: 0,1,1,1,]:}
\end{array}\right. \\
& \left.\begin{array}{l}
\left.-;-; \rho,-\frac{y}{v} ; \frac{x}{v} ; \frac{-w t}{1-t},-w, \frac{v t}{1-t}, w v\right] \\
-;-; 1+\beta ; 1+\alpha ;
\end{array}\right]
\end{aligned}
$$

(ii) Applying $\mathrm{p}=\mathrm{q}, \mathrm{a}_{\mathrm{j}}=\mathrm{b}_{\mathrm{j}}, \mathrm{j}=1,2, \ldots, \mathrm{p}$ and writting $w \rightarrow 0$ in (2.1), we get

$$
\begin{aligned}
& \sum_{n=0}^{\infty} \frac{(\rho)_{n}(n !)^{2}}{\left[(1+\alpha)_{n}\right]^{2}(1+\beta)_{n}} B_{n}^{(\alpha, \beta)}(x, y, v) L_{n}^{\alpha}(x) t^{n} \\
& =(1-t)^{-\rho} F_{2: 0 ; 0 ; 1 ; 1}^{1: 0 ; 0 ; 1}\left[\begin{array}{c}
{[\rho: 1,1,1,1] \quad:} \\
{[1+\alpha: 1,1,0,1],[\rho: 0,1,1,1,]:}
\end{array}\right. \\
& \left.\begin{array}{l}
-;-; \rho,-\frac{y}{v} ; \frac{x}{v} ; \frac{-x t}{1-t},-x, \frac{v t}{1-t}, x v \\
-;-; 1+\beta ; 1+\alpha ;
\end{array}\right]
\end{aligned}
$$

(iii) On taking $v \rightarrow 0$ in (2.1), we get

$$
\begin{gathered}
\sum_{n=0}^{\infty} \frac{(\rho)_{n}(n !)^{2}}{\left[(1+\alpha)_{n}\right]^{2}(1+\beta)_{n}} \times \\
L_{n}^{(\alpha, \beta)}{ }_{(x, y){ }_{n ; \lambda ;\left(b_{q}\right)}^{\alpha ; \mu ;\left(a_{p}\right)}(x, w) t^{n}}^{\text {nat }}
\end{gathered}
$$




$$
\begin{aligned}
& =(1-t)^{-\rho} F_{q+2: 0 ; 0 ; 0 ; 1 ; 1}^{p+2: 0 ; 0 ; 1 ; 1 ;}\left[\begin{array}{l}
{\left[\left(a_{p}\right): 1,1,1,0,1\right],} \\
{\left[\left(b_{q}\right): 1,1,1,0,1\right],}
\end{array}\right. \\
& {[\rho: 1,1,0,1,1],\left[\frac{x}{w}-\mu+1: 1,1,0,0,1\right]:} \\
& {[1+\alpha: 1,1,0,0,1],[\rho: 0,1,0,1,1,]:} \\
& \left.\begin{array}{l}
\left.-;-;-\frac{x}{w}+\lambda ; \rho ;-; \frac{-w t}{1-t},-w, w, \frac{-y t}{1-t}, w x\right] \\
-;-;-; 1+\beta ; 1+\alpha ;
\end{array}\right]
\end{aligned}
$$

(iv) By writing $\mu=1, \lambda=0, \mathrm{p}=\mathrm{q}$ and $\mathrm{a}_{\mathrm{j}}=\mathrm{b}_{\mathrm{j}}, \mathrm{j}=1$, $2, \ldots, \mathrm{p}$ and letting $v \rightarrow 0$, in (2.1), we have (2.6)

$$
\begin{aligned}
& \sum_{n=0}^{\infty} \frac{(\rho)_{n}(n !)^{2}}{\left[(1+\alpha)_{n}\right]^{2}(1+\beta)_{n}} \times \\
& L_{n}^{(\alpha, \beta)}{ }_{(x, y)} J_{n}^{\alpha}(x, w) t^{n} \\
& =(1-t)^{-\rho} F_{2: 0 ; 0 ; 1 ; 1}^{2: 0 ; 0 ; 1 ; 0}\left[\begin{array}{c}
{[\rho: 1,1,1,1],} \\
{[1+\alpha: 1,1,0,1],}
\end{array}\right. \\
& {\left[\frac{x}{w}: 1,1,0,1\right]:-;-; \rho ;-;} \\
& {[\rho: 0,1,1,1]:-;-; 1+\beta ; 1+\alpha \text {; }} \\
& \left.\frac{-w t}{1-t},-w, \frac{y t}{1-t}, w x\right]
\end{aligned}
$$

(v) Taking $v \rightarrow 0, w \rightarrow 0, \mathrm{p}=\mathrm{q}$ and $\mathrm{a}_{\mathrm{j}}=\mathrm{b}_{\mathrm{j}}, \mathrm{j}=1$, $2, \ldots, \mathrm{p}$ in $(2.1)$, we get

$$
\begin{gathered}
\sum_{n=0}^{\infty} \frac{(\rho)_{n}(n !)^{2}}{\left[(1+\alpha)_{n}\right]^{2}(1+\beta)_{n}} L_{n}^{(\alpha, \beta)}{ }_{(x, y)} L_{n}^{\alpha}(x) t^{n} \\
=(1-t)^{-\rho} F_{2: 0 ; 0 ; 1 ; 1}^{1: 0 ; 0 ; 1 ; 0[}[1+\alpha: 1,1,0,1],[\rho: 0,1,1,1]: \\
\left.-;-; \rho ;-; \quad \frac{-x t}{[\rho t},-x, \frac{y t}{1-t}, x^{2}\right] \\
-;-; 1+\beta ; 1+\alpha ; 1-t
\end{gathered}
$$

(vi) Taking $\beta=0, \mathrm{y}=0 \quad$ in (2.7), we get

$$
\begin{gathered}
\sum_{n=0}^{\infty} \frac{(\rho)_{n}(n !)}{\left[(1+\alpha)_{n}\right]^{2}} L_{n(x)}^{\alpha} L_{n}^{\alpha}(x) t^{n} \\
=(1-t)^{-\rho} F_{2: 0 ; 0 ; 1}^{1: 0 ; 0 ; 0}\left[\begin{array}{c}
{[\rho: 1,1,1] \quad:} \\
{[1+\alpha: 1,1,1],[\rho: 0,1,1]:}
\end{array}\right.
\end{gathered}
$$

$$
\begin{aligned}
& -;-;-; \\
& -;-; 1+\alpha \\
& \left.-\frac{-x t}{1-t},-x, x^{2}\right]
\end{aligned}
$$

These are all the bilateral (bilinear) generating relations for the class of generalized hypergeo-metric functions (1.1), whereas the results for the modified Jacobi polynomial, Laguerre poly-nomial of two variables and Laguerre poly-nomial are believed to be new.

Proof of (2.2). The result (2.2) can also be deduced by using (1.9) and the same techniques as followed in the previous result.

(i). By letting limit $v \rightarrow 0$ in (2.2), we obtain

$$
\begin{aligned}
& \sum_{n=0}^{\infty} \frac{(n !)^{2}}{(1+\alpha)_{n}(1+\beta)_{n}} L_{n}^{(\alpha, \beta)}{ }_{(x, y)} \phi_{n}(x ; \lambda) t^{n} \\
& =(1-t)^{x}\left(1-t e^{-\lambda}\right)^{-x-1} F_{2: 0 ; 0 ; 1}^{2: 0 ; 0 ; 0}\left[\begin{array}{c}
{[1: 1,1,1],} \\
{[1+\beta: 1,1,1],}
\end{array}\right. \\
& \left.\begin{array}{l}
{[-x: 1,0,1]:-;-;-;} \\
{[1: 1,0,1]:-;-; 1+\alpha ; t_{1}, t_{2}, t_{3}}
\end{array}\right]
\end{aligned}
$$

where

$$
\begin{gathered}
t_{1}=\frac{y\left(e^{\lambda}-1\right) t}{\left(e^{\lambda}-t\right)(1-t)}, t_{2}=\frac{-y t}{e^{\lambda}-t}, \\
t_{3}=\frac{y x\left(e^{\lambda}-1\right) t}{\left(e^{\lambda}-1\right)(1-t)} .
\end{gathered}
$$

is the bilateral generating relation for the Laguerre polynomial of two variables, which is believed to be new. Proof of (2.3). The result (2.3) can also be deduced by using (1.10) and the same techniques as followed in the result (2.1).

Application. Applying limit $v \rightarrow 0$ on (2.3), we obtain the result

$$
\begin{gathered}
\sum_{n=0}^{\infty} \frac{(n !)^{2}}{(1+\alpha)_{n}(1+\beta)_{n}} L_{n}^{(\alpha, \beta)}(x, y) f_{n}(x ; a) t^{n} \\
=(1-t)^{-x} e^{a x t} F_{1: 0 ; 0 ; 1}^{1: 0 ; 0 ; 0}\left[\begin{array}{c}
{[x: 1,0,1]:} \\
{[1+\beta: 1,1,1],-:}
\end{array}\right. \\
\left.-;-; \frac{x}{v} ;\left(\frac{-y t}{1-t}\right)^{n}, y \operatorname{tax},\left(\frac{-x y t}{1-t}\right)\right] \\
-;-; 1+\alpha ;
\end{gathered}
$$

is the bilateral generating relation for the Laguerre polynomial of two variables, which is believed to be new. 


\section{Conclusion}

By employing the technique used in the proof of (2.1) and adjusting the parameters one can easily get the bilateral generating relations.

\section{Acknowledgements}

The authors are thankful to the referee for giving some useful suggestions to improve this paper.

\section{REFERENCES}

[1] A. K. Agarwal and H. L. Manoch. On some new generating functions, Inadian J. Pure and Appl. Math., Vol. 13, No. 11, 1369-1375, 1982..

[2] Konhouser, D.E. Joseph, Biorthogonal polynomial suggested by the Laguerre polynomial, Pacific J. Math. Vol. 21, 303-314, 1967.

[3] M. Lahiri and B. Satyanarayana, A class of generalized hypergeometric functions defined by using a difference operator, Soochow J. Math. Vol. 19,, 163-171, 1993.

[4] M. Lahiri and B. Satyanarayana, Certain bilateral generating relations for generalized hypergeometric functions, Proc. Indian Acad. Sci. (Math Sci.), Vol. 105, No. 3, 297-301, 1995.

[5] M. Lahiri and B. Satyanarayana, Discrete and pseudo orthogonality for a class of generalized hypergeometric functions, Tamkong J. of Math. Vol. 28, No. 4, 325-332, 1997.

[6] C. L. Parihar and V. C. Patel, On modified Jacobi polynomials, J. Indian Acad. Math. Vol. 1, 41-46, 1979.

[7] S. F. Ragab, On Laguerre polynomial of two variables , Bull. Cal. Math. Soc. Vol. 83, 253-262, 1991.

[8] E. D.. Rainville, Special Functions, Macmillan, New York, 1960.

[9] B.Satyanarayana, Discrete hypergeo-metric functions defined by a difference operator technique, Ph.D. thesis, Banaras Hindu University, Varanasi, India, 1993.

[10] B. Satyanarayana, N. Srimannarayana and Y. Pragathi Kumar, A class of generalized hypergeometric functions of two discrete variables, Analele Universitatii Constantin Brancusi Din Targu Jiu, Seria Inginerie,Vol. 3, 260-269, 2010.

[11] H. M. Srivastava and P. W. Karlesson, Multiple Gaussian Hypergeometric Series, Halsted Press (Ellis Horwood Limited Chichester) John Wiley and Sons, New York, 1985. 